\title{
Identification of long non-coding RNAs as novel biomarker and potential therapeutic target for atrial fibrillation in old adults
}

\author{
Yingjia $X_{\mathbf{u}^{1}}$, Ritai Huang${ }^{2}$, Jianing $\mathbf{G u}^{3}$ and Weifeng Jiang ${ }^{1}$ \\ ${ }^{1}$ Department of Cardiology, Shanghai Chest Hospital, Shanghai Jiao Tong University, Shanghai, China \\ ${ }^{2}$ Department of Cardiothoracic Surgery, Ren Ji Hospital, School of Medicine, Shanghai JiaoTong University, Shanghai, China \\ ${ }^{3}$ Department of Cardiology, Rui Jin Hospital, School of Medicine, Shanghai Jiao Tong University, Shanghai, China \\ Correspondence to: Yingjia Xu, email: shdrxu@hotmail.com \\ Ritai Huang, email: ihgroup@126.com \\ Keywords: Iong non-coding RNAs (InCRNAs), atrial fibrillation (AF), transcription factors, Gerotarget \\ Received: September 13,2015 Accepted: February 05, $2016 \quad$ Published: February 18, 2016
}

\section{ABSTRACT}

Atrial fibrillation (AF) is a highly prevalent cardiac arrhythmia disease, which widely leads to exacerbate heart failure and ischemic stroke in elder world. Recently, long non-coding RNAs (IncRNAs), a subclass of noncoding RNAs, have been reported to play critical roles in pathophysiology of cardiac heart. However, little is known of their role in cardiac arrhythmia. In the present study, we investigated the expression levels of IncRNAs of AF patients and healthy people with Agilent Human IncRNA array for the first time. 177 IncRNAs of 78243 and 153 mRNAs of 30215 tested were identified to be differentially expressed ( $\geq 2$-fold change), indicating that the expression of many IncRNAs are upregulated or downregulated in AF. Among these, NONHSAT040387 and NONHSAT098586 were the most upregulated and downregulated IncRNAs. Real time quantitative PCR were employed to validate the microarray analysis findings, and the results confirmed the consistence. GO and KEGG pathway analysis were applied to explore the potential IncRNAs functions, some pathways including oxygen transporter activity and protein heterodimerization activity were speculated to be involved in AF pathogenesis. These results shed some light on IncRNAs' physiologic functions and provide useful information for exploring potential therapeutic treatments for heart rhythm disease.

\section{INTRODUCTION}

Atrial fibrillation (AF) is the most common heart rhythm disease in the world, accounting approximately $0.5 \%$ of the total world population $[1,2]$. AF exacerbates heart failure and ischemic stroke, substantially increases the morbidity and mortality, resulting in a higher burden for patients and even the nations. However, a higher incidence of adverse consequences for the elderly has been associated with atrial fibrillation. Although $\mathrm{AF}$ is a heterogeneous disease, previous reports suggest that the arrhythmia may arise due to the interaction by genetic and acquired risk factors - the so-called "double hit" hypothesis [3]. Unfortunately, the precise mechanisms of atrial remodeling were not well elucidated, leading to the demand of investigating the exact mechanisms of the disease and developing treatments. Identification of novel biomarker influencing the development of AF is critical to the understanding and future prevention of the disease. Long non-coding RNAs (lncRNAs) are a subclass of noncoding RNAs (ncRNAs) and are transcribed from the genome with at least 200 nucleotides [4, 5]. The ncRNAs include microRNAs (miRNAs), PIWI interacting RNAs, and endogenous small interfering RNAs. In fact, the notion of ncRNAs acting as heart disease modulators is not new; reviews regarding the role of ncRNAs in heart disease have already been published before [6]. It has become increasingly apparent that many of the lncRNAs play molecular functions, such as controlling cell cycle, differentiation, apoptosis or as smaller RNA precursors [6-8]. LncRNAs may function through a variety of mechanisms such as modulating gene transcription by rearranging chromosomal looping and transcription factor binding. LncRNAs also affect miRNA functions 
Table 1: Top 25 aberrantly expressed IncRNAs in microarray for three pairs of AF and normal blood

\begin{tabular}{|c|c|c|c|c|c|c|c|c|c|c|c|c|}
\hline TargetID & FC (abs) & $\mathbf{p}$ & Regulation & N1 & N2 & N3 & AF1 & AF2 & AF3 & $\mathrm{Chr}$ & start & end \\
\hline NONHSAT098586 & 7.51 & 0.01 & up & 2.70 & 4.54 & 3.94 & 6.32 & 6.76 & 6.83 & chr4 & 144918491 & 144940455 \\
\hline NONHSAG007503 & 7.34 & 0.02 & up & 12.37 & 12.85 & 10.51 & 14.53 & 14.51 & 15.32 & chr11 & 5246697 & 5248302 \\
\hline NONHSAT040387 & 6.94 & 0.01 & down & 6.73 & 6.78 & 7.11 & 2.87 & 4.72 & 4.66 & chr14 & 106068035 & 107099427 \\
\hline NONHSAT076398 & 6.80 & 0.00 & up & 3.80 & 2.82 & 2.83 & 6.03 & 5.92 & 5.78 & chr2 & 202316456 & 202323546 \\
\hline NONHSAT053927 & 6.77 & 0.01 & up & 3.29 & 4.28 & 3.13 & 5.91 & 6.07 & 7.01 & $\operatorname{chr} 17$ & 42338172 & 42345509 \\
\hline NONHSAT098582 & 6.55 & 0.03 & up & 4.55 & 5.13 & 2.63 & 6.81 & 6.40 & 7.23 & chr4 & 144917349 & 144940477 \\
\hline NONHSAT040421 & 6.52 & 0.03 & down & 5.58 & 6.21 & 6.63 & 2.78 & 4.88 & 2.66 & chr14 & 106329999 & 107034966 \\
\hline NONHSAT017667 & 6.00 & 0.02 & up & 15.01 & 15.47 & 13.18 & 17.10 & 16.87 & 17.43 & chr11 & 5246884 & 5248053 \\
\hline TCONS_12_00030433 & 5.25 & 0.00 & down & 7.66 & 8.28 & 8.29 & 5.06 & 5.70 & 6.29 & $\operatorname{chrX}$ & 3820106 & 3855883 \\
\hline NONHSAT039492 & 5.21 & 0.01 & down & 10.72 & 10.45 & 9.85 & 7.62 & 8.70 & 7.57 & chr14 & 96177059 & 96178299 \\
\hline NONHSAT090718 & 5.15 & 0.04 & up & 5.35 & 3.08 & 3.59 & 6.91 & 6.67 & 5.53 & chr3 & 93624883 & 93630001 \\
\hline NONHSAG053956 & 5.03 & 0.00 & down & 7.97 & 8.40 & 8.26 & 5.63 & 5.64 & 6.36 & $\operatorname{chrX}$ & 3735557 & 3785832 \\
\hline NONHSAT098591 & 4.92 & 0.00 & up & 4.05 & 3.48 & 3.56 & 5.95 & 5.77 & 6.26 & chr4 & 145035828 & 145061788 \\
\hline NONHSAT136136 & 4.81 & 0.00 & down & 7.29 & 7.70 & 7.72 & 4.75 & 5.16 & 6.01 & $\operatorname{chr} \mathrm{X}$ & 3735579 & 3785832 \\
\hline XR_245045.1 & 4.57 & 0.05 & down & 6.44 & 6.89 & 8.40 & 4.12 & 5.71 & 5.34 & & & \\
\hline NONHSAT141981 & 4.45 & 0.00 & down & 7.40 & 7.30 & 7.92 & 4.88 & 5.95 & 5.33 & chr16 & 31973408 & 31985682 \\
\hline NONHSAT142052 & 4.42 & 0.02 & down & 5.80 & 5.91 & 6.57 & 3.03 & 4.67 & 4.16 & chr16 & 32926394 & 32926857 \\
\hline NONHSAT076870 & 4.35 & 0.05 & up & 6.19 & 5.47 & 3.85 & 7.11 & 6.90 & 7.87 & chr2 & 218678431 & 218680091 \\
\hline NONHSAT040390 & 4.32 & 0.00 & down & 10.72 & 10.84 & 11.13 & 8.30 & 9.24 & 8.81 & chr14 & 106111027 & 106478375 \\
\hline ENST00000460164 & 4.18 & 0.03 & down & 13.24 & 12.64 & 13.65 & 9.98 & 11.83 & 11.51 & 14 & 106110833 & 106114892 \\
\hline ENST00000456563 & 4.10 & 0.00 & down & 8.76 & 9.10 & 9.22 & 6.43 & 7.09 & 7.46 & $\mathrm{X}$ & 3772390 & 3785176 \\
\hline NONHSAT040540 & 4.02 & 0.02 & down & 6.48 & 7.04 & 7.05 & 4.03 & 5.79 & 4.73 & chr14 & 107108251 & 107108696 \\
\hline NONHSAT076401 & 3.98 & 0.00 & up & 5.85 & 5.87 & 5.55 & 7.86 & 7.45 & 7.95 & chr2 & 202343864 & 202345071 \\
\hline NONHSAT136135 & 3.98 & 0.00 & down & 7.64 & 8.26 & 8.03 & 5.62 & 6.10 & 6.23 & $\operatorname{chr} \mathrm{X}$ & 3735575 & 3742639 \\
\hline NONHSAT055019 & 3.95 & 0.03 & up & 4.35 & 2.68 & 2.68 & 5.42 & 4.76 & 5.47 & $\operatorname{chr} 17$ & 57695918 & 57696926 \\
\hline
\end{tabular}

by controlling pre-mRNA splicing or as miRNA sponges. Recently, accumulating evidences indicate that there is aberrant expression of lncRNAs in many heart diseases, including heart failure, pathological hypertrophy and Ventricular Septal Defect etc [9-11]. However, to the best of our knowledge, no attempts have been made to investigate the possible involvement of IncRNAs expression in $\mathrm{AF}$, and the underlying pathways remain poorly understood.

\section{RESULTS}

\section{LncRNAs and mRNAs expression profiles in AF}

LncRNA profiling showed 177 lncRNAs of 78243 tested with significant differential expression levels at least a two-fold change in AF patients compared with normal patients, with 100 up-regulated and 77 down-regulated, respectively. The top 25 differentially expressed lncRNAs were listed in Table 1. Among the dysregulated lncRNA transcripts, NONHSAT098586 is the most up-regulated, with a fold of change (FC) of 7.51, whereas NONHSAT040387 is the most down- regulated, FC being 6.94. Using the same criteria as the lncRNAs, we found that 75 up-regulated and 78 downregulated mRNA transcripts. The most up-regulated and downregulated mRNA transcripts are hemoglobin gamma A (NM_000559) and desmoplakin (NM_004415), with FCs of 16.03 and 14.84, respectively (shown in Table 2). Hierarchical clustering of the lncRNA and mRNA profiles was performed using cluster 3.0.2; Hierarchical clustering of the expression of the 177 lncRNAs based on centered Pearson correlation clearly separated AF from normal control (Figure 1).

\section{Validate the results of microarray by $q$ PCR}

LncRNAs transcripts were validated by quantitative PCR with 30 human blood samples (20 AF samples and 10 control samples). Two lncRNAs (NONHSAG007503 and NONHSAT040387) were randomly selected to prove the consistency of microarray and qPCR. As expected, the expression of lncRNA NONHSAG007503 was upregulated and NONHSAT040387 was down-regulated in the AF samples versus control samples (Figure 2), consistent with the microarray results. 


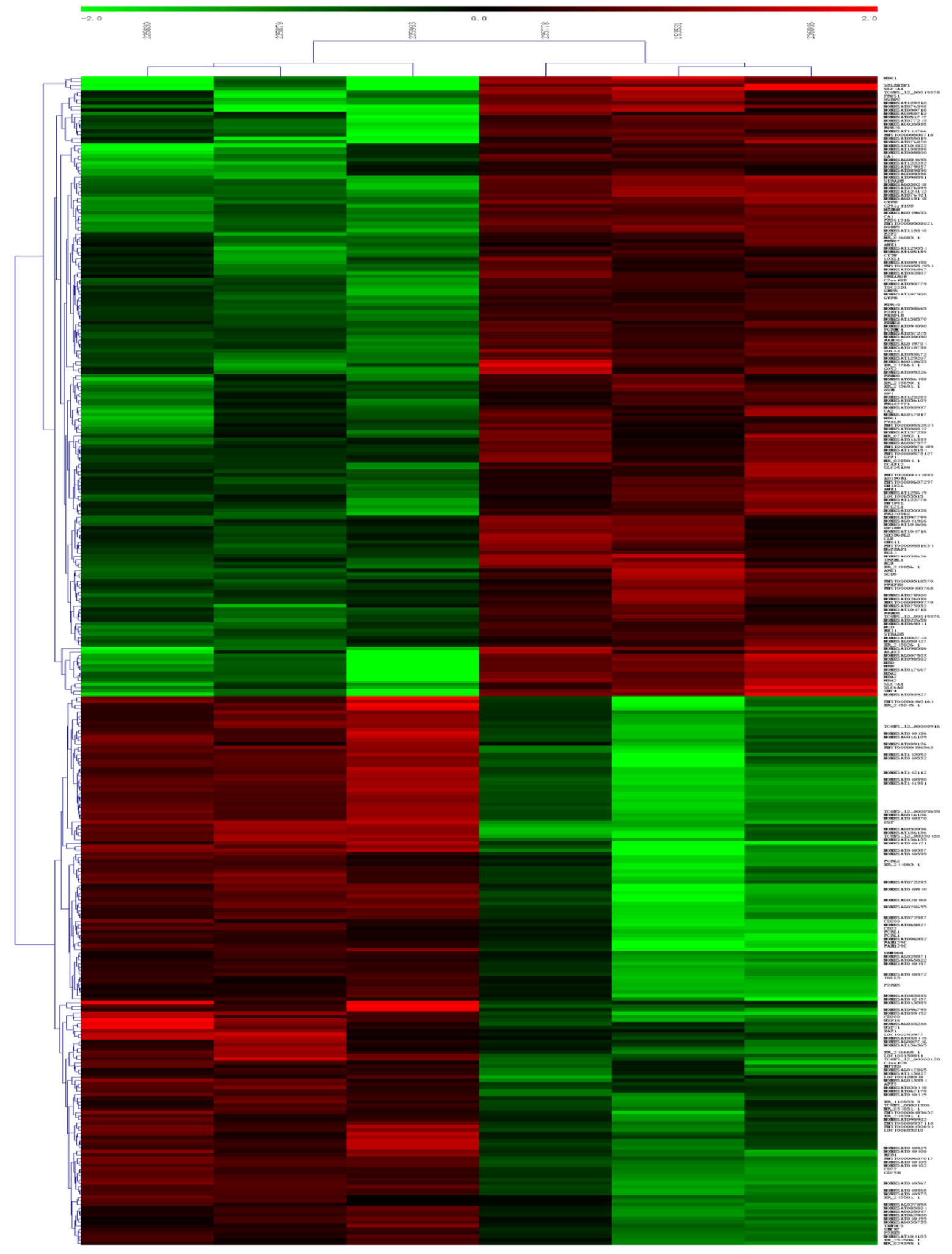

Figure 1: Heat map and hierarchical clustering of IncRNA profile comparison between the AF and normal blood samples. Each row represents one lncRNA, and each column represents one sample. The relative lncRNA expression is depicted according to the color scale. Red indicates up-regulation; green indicates downregulation. 2.0 and -2.0 are fold changes in the corresponding spectrum, whereas left represents normal samples and right represents AF samples. The differentially expressed lncRNAs are clearly self-segregated into clusters. 
(A).

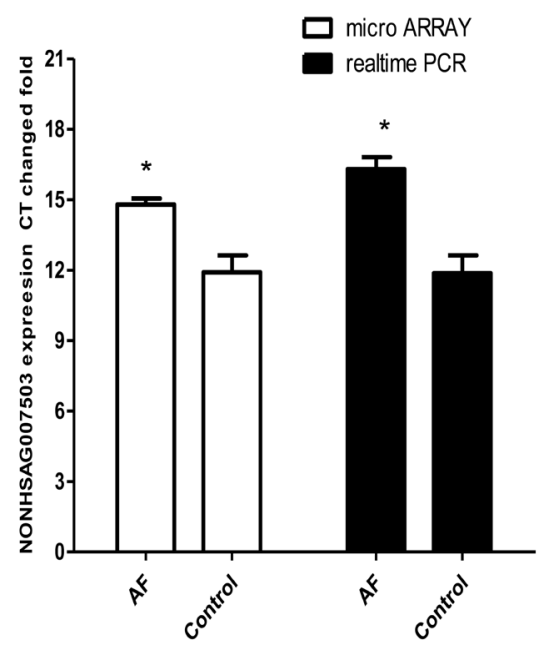

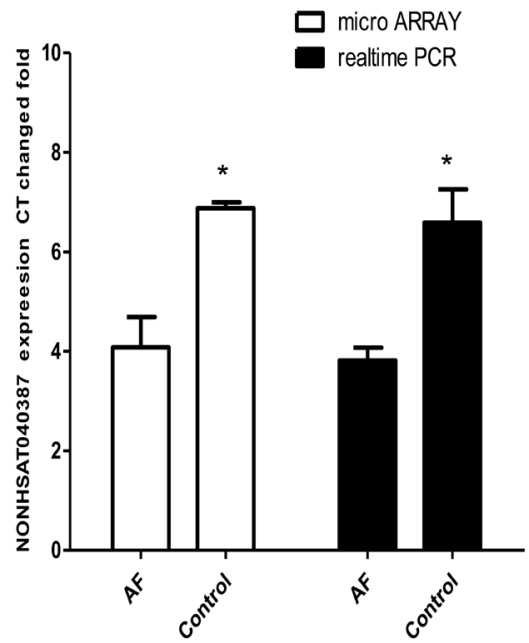

Figure 2: Comparisons between microarray data and qPCR results. NONHSAG007503 A. and B. NONHSAT040387 were differentially expressed in AF samples compared with control samples detected by microarray and real-time quantitative PCR. The validation results of the lncRNAs indicated that the microarray data correlated well with the qPCR results. The results are presented as mean $\pm \operatorname{SEM}\left(n=3\right.$ in micro array, $n=10$ in realtime PCR). ${ }^{*} p<0.05$ and $* * p<0.01$ vs corresponding controls.

(A). GO analysis

Function

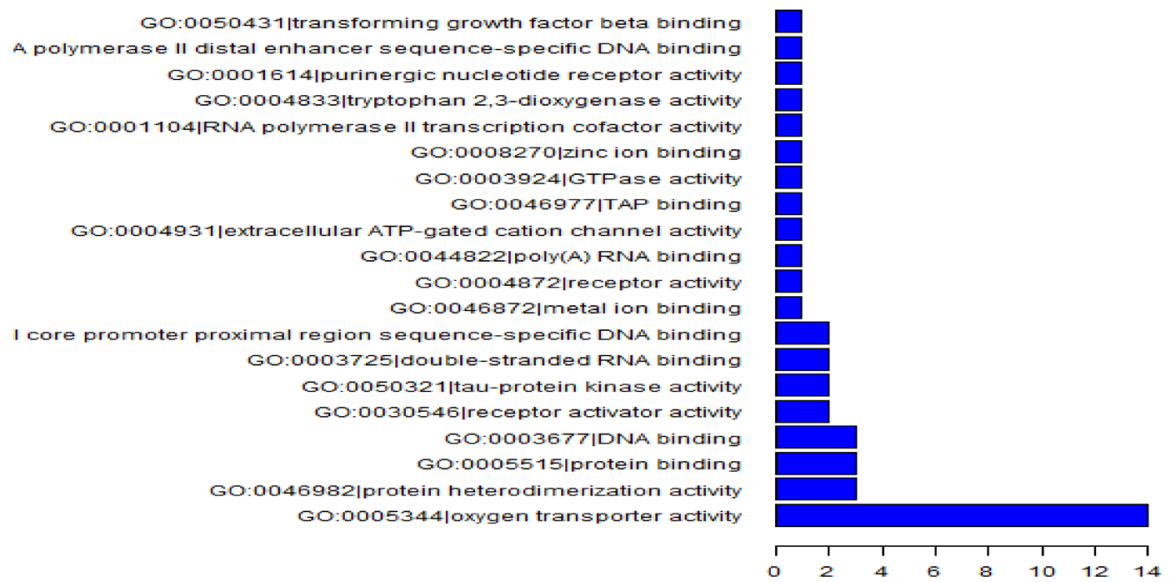

(B). KEGG Pathway analysis

enrich_stat

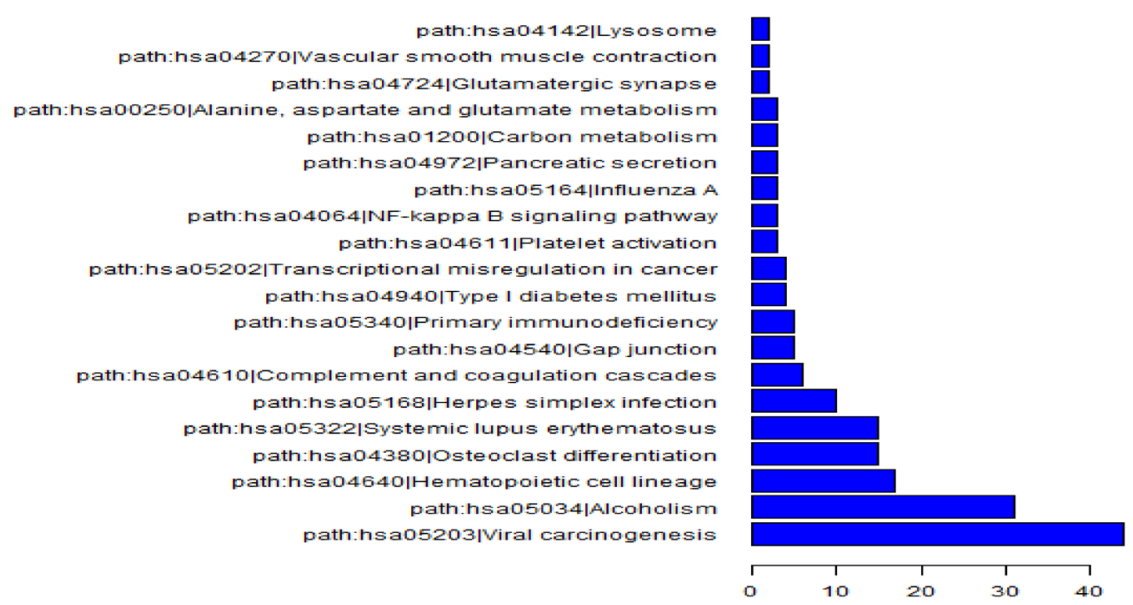

Figure 3: GO analysis A. and KEGG Pathway analysis B. of aberrantly expressed lncRNAs in AF. 


\section{Go and pathway analysis}

To predict the functions of the lncRNAs, we adopted methods originally demonstrated in previous reported paper [12]. Generally, The GO category was classified by Fisher's exact test, and the $p$-value was corrected by the false discovery rate (FDR) calculation. The presenting key genes in gene networks and canonical pathways were identified by the curated Ingenuity Pathway Analysis (IPA) database according to KEGG. The enriched functional terms were used as the predicted functional terms for each given lncRNAs. GO analysis indicated that several functional pathways were enriched. Among these pathways, oxygen transporter activity, protein heterodimerization activity, and DNA binding were the most closely associated with AF (Figure 3A). Furthermore, using the same criteria as the GO analysis,
KEGG Pathway analysis showed some corresponding pathways, including viral carcinogenesis, alcoholism, hematopoietic cell lineage, osteoclast differentiation and complement and coagulation cascades, etc. (Figure 3B).

\section{Construction of co-expression network}

To explore which lncRNAs and mRNAs play critical roles in $\mathrm{AF}$ progression, we constructed a co-expression network of the differentially expressed correlated lncRNAs and mRNAs. The correlation between lncRNAs and mRNAs was expressed with Pearson's correlation coefficients and those no less than 0.99 were used to construct the network. Transcriptional regulatory elements may exist in non-coding regions, but it could be tough to distinguish these only guiding by primary sequences. To explore lncRNAs that possibly have trans-regulating

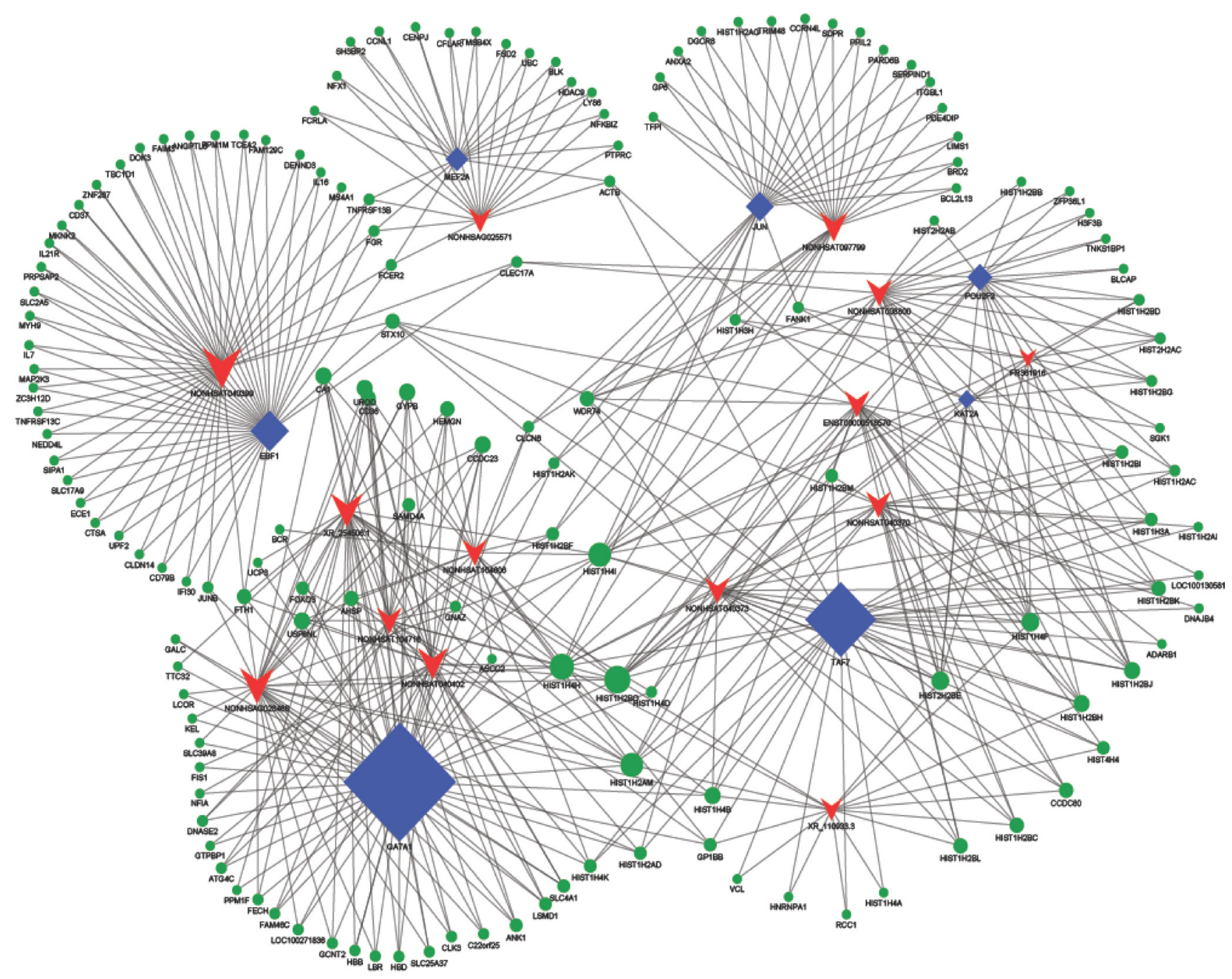

Figure 4: LncRNA-mRNA-network was constructed based on the correlation analysis between the differential expressed IncRNAs and mRNAs. In the network, blue node represents Transcription factors, red node represents the lncRNAs, and green node represents the target mRNAs. The size of node is proportional to the outgoing link number. The thickness of outgoing link represents statistical relationship to the number of occurrences of the results proportionally. 
Table 2: Top 25 aberrantly expressed mRNAs in microarray for three pairs of AF and normal blood

\begin{tabular}{|c|c|c|c|c|c|c|c|c|c|c|}
\hline $\begin{array}{l}\text { Genbank } \\
\text { Accession } \\
\end{array}$ & Gene Name & FC (abs) & $\mathbf{p}$ & Regulation & N1 & N2 & $\mathbf{N 3}$ & AF1 & AF2 & AF3 \\
\hline NM_000559 & HBG1 & 16.03 & 0.01 & up & 4.97 & 7.33 & 4.25 & 9.98 & 9.40 & 9.18 \\
\hline NM_000342 & SLC4A1 & 14.84 & 0.00 & up & 3.15 & 4.55 & 2.77 & 7.31 & 6.75 & 8.09 \\
\hline NM_003944 & SELENBP1 & 9.25 & 0.02 & up & 4.31 & 5.50 & 2.77 & 6.99 & 6.99 & 8.22 \\
\hline DQ656067 & clone Affy2H6-6 & 8.66 & 0.02 & up & 4.87 & 6.87 & 4.49 & 8.68 & 8.77 & 8.11 \\
\hline NM_000519 & HBD & 6.24 & 0.02 & up & 14.37 & 15.04 & 12.74 & 16.55 & 16.53 & 16.99 \\
\hline NM_000517 & HBA2 & 6.15 & 0.02 & up & 13.82 & 13.80 & 11.72 & 15.40 & 15.75 & 16.05 \\
\hline NM_004415 & DSP & 6.09 & 0.00 & down & 5.35 & 5.51 & 5.75 & 2.93 & 3.09 & 2.77 \\
\hline XM_003119591 & uncharacterized LOC100508797 & 5.80 & 0.04 & down & 7.52 & 6.98 & 7.42 & 3.18 & 5.75 & 5.39 \\
\hline NM_007308 & SNCA & 5.76 & 0.01 & up & 10.48 & 10.84 & 9.37 & 12.80 & 12.44 & 13.04 \\
\hline NM_000518 & HBB & 5.75 & 0.03 & up & 15.36 & 15.64 & 13.28 & 17.22 & 17.09 & 17.54 \\
\hline NM_000032 & ALAS2 & 4.88 & 0.04 & up & 6.45 & 8.03 & 5.64 & 8.44 & 9.55 & 8.99 \\
\hline AY172958 & $\begin{array}{l}\text { anti-rabies SOJB immunoglobulin heavy } \\
\text { chain }\end{array}$ & 4.87 & 0.03 & down & 10.42 & 10.10 & 11.64 & 7.39 & 9.17 & 8.75 \\
\hline NM_017414 & USP18 & 4.60 & 0.01 & down & 7.63 & 6.71 & 6.10 & 4.15 & 5.01 & 4.67 \\
\hline HCG1981372 & & 4.29 & 0.00 & down & 8.17 & 8.80 & 8.76 & 6.25 & 6.25 & 6.95 \\
\hline XM_003403505 & Ig kappa chain V-III region VH-like & 4.27 & 0.01 & down & 9.61 & 9.56 & 9.87 & 6.65 & 8.18 & 7.93 \\
\hline AY505570 & Immunoglobulin heavy chain & 4.22 & 0.00 & down & 9.56 & 9.36 & 10.13 & 7.16 & 8.09 & 7.57 \\
\hline A_33_P3331178 & & 4.21 & 0.02 & down & 7.63 & 7.74 & 8.20 & 5.28 & 6.77 & 5.29 \\
\hline A_24_P110242 & & 3.99 & 0.03 & down & 9.48 & 9.72 & 10.90 & 7.39 & 8.80 & 7.93 \\
\hline NM_002100 & GYPB & 3.93 & 0.00 & up & 3.44 & 3.56 & 3.67 & 5.33 & 5.53 & 5.73 \\
\hline XM_003403854 & Ig heavy chain V-III region VH26-like & 3.92 & 0.01 & down & 10.49 & 10.46 & 10.82 & 7.74 & 9.06 & 9.06 \\
\hline XM_003403466 & Ig heavy chain V-I region V35-like & 3.86 & 0.01 & down & 11.01 & 10.85 & 11.47 & 8.56 & 9.63 & 9.30 \\
\hline Z46771 & Immunoglobulin heavy chain VDJ region & 3.85 & 0.00 & down & 9.15 & 9.07 & 9.73 & 6.94 & 7.87 & 7.31 \\
\hline L03830 & Ig rearranged H-chain mRNA V-region & 3.84 & 0.01 & down & 8.62 & 8.70 & 8.86 & 6.21 & 7.50 & 6.66 \\
\hline Z18824 & Rearranged Ig H-chain V-domain & 3.83 & 0.01 & down & 8.56 & 8.75 & 9.56 & 6.46 & 7.65 & 6.95 \\
\hline AY393117 & $\begin{array}{l}\text { Clone RA702-M1-22.fa Immunoglobulin } \\
\text { heavy chain }\end{array}$ & 3.78 & 0.01 & down & 9.71 & 9.68 & 10.13 & 7.40 & 8.51 & 7.85 \\
\hline
\end{tabular}

functions, we compared the mRNAs that coexpressed with these lncRNAs with those mRNAs including regulatory targets of certain Transcription factors (TFs). It can be helpful to accurately map the boundaries of regulatory elements because of the narrow transcription factor binding sites. As shown in Figure 4, GATA1 closely correlated with many mRNAs and lncRNAs. Similar results were showed in TAF7 and EBF1, indicating the three transcriptional regulatory elements may play critical roles in lncRNAs process.

\section{DISCUSSION}

$\mathrm{AF}$ is the most common cardiac diseases, bringing huge burdens to old patients and their families. The prevalence of AF increases rapidly with age. However, the exact pathogenesis and serum biomarkers of $\mathrm{AF}$ are still not well elucidated. Sensitive serum biomarkers reflect the development of atrial remodeling, thus it could be simpler to determine the state of atrial remodeling and perform interventional therapy timely through observing serum biomarkers [13]. However, so far the correlation among lncRNAs, atrial remodeling and serum biomarkers remains unknown. To the best of our knowledge, this study is the first comprehensive lncRNAs analysis with AF in blood. This study was designed to discover the relationship between lncRNAs expression and atrial structural remodeling of AF. These data indicated that 177 lncRNAs displayed significant differential expression in AF. Functional elements are always identified by extreme conservative evolutionary sequences, but many lncRNAs are poorly conserved though they play roles in the heart. Ruan et al has discovered 219 lncRNAs differentially expressed in atrial tissues between AFs and controls [14]. Our study results are consisted with Ruan's study. For example, GO analysis found same changes in molecular function between AF compared with controls, including DNA binding, protein binding, metal ion binding and transforming growth factor beta binding. Differential expression of lncRNAs may be affected by $\mathrm{AF}$ and atrial remodeling and our work has identified the differentially expressed lncRNAs in AF. However, it needs further investigation to confirm the relationship between their expression and function. Subgroup analysis of IncRNAs should be performed to further exploration on the regulatory network. Unfortunately, available experimentally verified lncRNA associations are still 
comparatively rare, further functional studies are required to elucidate their roles in AF. These findings bring profound influences on cardiovascular science research and provide golden opportunities for intervention therapies in disease progression.

Cardiac science including development and adaptation regulatory networks has been intensively investigated. In the past ten years, with the help of development in molecular and biotechnology, scientists have achieved great progress in elucidating the molecular mechanisms of heart formation. In recent years, genetics research based on family and population showed that transcription factors may play important roles in arrhythmia susceptibility [14-16]. Moreover, animal studies have demonstrated that transcription factors performed crucial roles in atrial remodeling, indicating the importance of transcription factors in AF. In the present study, we found that several transcription factors such as GATA1, TAF7 and EBF1 could be essential for lncRNAs expression in AF development. Pathway identification showed that GATA1, TAF7 and EBF1 played central roles in $\mathrm{AF}$, which were consistent with previous reports $[17,18]$. Close physical links between lncRNAs and developmental functional genes does not necessarily indicate that there would be functional links between protein-coding genes and lncRNAs. For example, recent researches on mice suggested that there were no evident correlations between expression levels of lncRNAs and their adjacent genes [19-22]. Therefore, further researches are demanded to fully elucidate the molecular mechanisms between these transcription factors and AF pathogenesis. In the future, such researches can help making significant progress in the clinical treatment of arrhythmia.

In conclusion, we explored and found out the dysregulated expression of IncRNAs in human AF for the first time. These data suggest that a great variety of lncRNAs are involved in AF development and present background/reference resources for future exploring the functions of lncRNAs in AF development. More investigation will be required to define the physiologic functions and the mechanisms by which these lncRNAs affecting AF formation.

\section{MATERIALS AND METHODS}

\section{Ethics statement}

This research was permitted by the human ethics committee of the Shanghai Chest Hospital, People's Republic of China. All AF patients and health control people have been informed with written consent to use their blood samples for this study. The AF patients were elder adults (age $=55 \pm 5$ years), without hypertension, diabetes, hyperthyroidism and other heart disease.

\section{Blood collection and RNA extraction}

$5 \mathrm{ml}$ blood of each person was collected and immediately stored at $4{ }^{\circ} \mathrm{C}$ until use. According to the manufacturer's protocols, RNA Isolation Kit (Ambion, USA) was used to extract total RNA from blood samples within $24 \mathrm{~h}$ after collection. Total RNA was quantified with the NanoDrop ND-2000 (Thermo Scientific, USA) and the RNA integrity was assessed using Agilent Bioanalyzer 2100 (Agilent Technologies, USA).

\section{LncRNA and mRNA microarray expression profiling}

Agilent Human LncRNA Microarray v 4.0 is designed for the analysis of global human lncRNAs and protein-coding transcripts. The microarray profiling was conducted in the laboratory of the OE Biotechnology Company in Shanghai, People's Republic of China. The sample labeling, microarray hybridization and washing were performed as described by the manufacturer. In brief, total RNA were transcribed to cDNA, synthesized into cRNA embedding with Cyanine-3-CTP. These tagged cRNAs were hybridized onto the Human IncRNA array, including a global profiling of 78,243 human lncRNAs and 30,215 coding transcripts. The arrays were scanned with the Agilent Scanner G2505C (Agilent Technologies, USA) after washing. Array images were analyzed by Feature Extraction software (version 10.7.1.1, Agilent Technologies) and the raw data were extracted. The raw data were further analyzed by Genespring (Version 12.5, Agilent Technologies). The raw data were firstly normalized, setting a change threshold $>2.0$ and $p$ value $<0.05$ for up- and down-regulated genes. Then, Hierarchical Clustering was employed to calculate the distinguishable lncRNA and mRNA expression patterns.

\section{Functional group analysis}

The functions in biological pathways or GO terms of these closest coding genes were analyzed by Pathway and GO analyses Kyoto Encyclopedia of Genes and Genomes (KEGG) analysis according to the latest KEGG database (http://www.genome.jp/kegg/) was employed to determine the biological roles of these differentially expressed mRNAs. Significance is judged when $p$ value (Hypergeometric-P value) is less than 0.05 .

\section{Co-expression network construction}

To discover the potential targets of IncRNA, we analyzed the interaction between lncRNAs and corresponding transcription factors based on hypergeometric cumulative distribution function with the 
help of MATLAB 2012b (The MathWorks, USA). The graph of the lncRNAs-TFs network was drawn with the help of Cytoscape 3.01 (Agilent and IBS, USA). If the intersection of these two groups is large enough $(p<0.01$, calculated by hypergeometric cumulative distribution function and FDR $<0.01$, under the control of the Benjamini and Hochberg procedure), then we predict that these lncRNAs possibly participate in pathways regulated by these TFs. The recently released ENCODE data on TFs and their regulatory targets were used in our analysis

\section{Real-time quantitative reverse transcription PCR}

A two-step reaction process was used for quantification reverse transcription [21] and PCR. Each RT reaction consisted of $0.5 \mu \mathrm{g}$ RNA, $2 \mu \mathrm{L}$ of Primer Script Buffer, $0.5 \mu \mathrm{L}$ of oligo dT, $0.5 \mu \mathrm{L}$ of random 6 mers, $0.5 \mu \mathrm{L}$ of Primer Script RT Enzyme Mix I (TaKaRa, Japan) and nuclease-free water to reach a volume of 10 $\mu \mathrm{L}$. Reactions were performed in the GeneAmp ${ }^{\circledR}$ PCR System 7500 (Applied Biosystems, USA) for $15 \mathrm{~min}$ at $37{ }^{\circ} \mathrm{C}$, then inactivation of RT by heating at $85{ }^{\circ} \mathrm{C}$ for $5 \mathrm{~s}$. Then the RT mix was diluted by 10 -fold with nuclease-free water and stored at $-20^{\circ} \mathrm{C}$. While running real-time quantitative PCR, melting curve was analyzed to verify the specificity of the aimed PCR product. All experiments were done in triplicate. Glyceraldehyde-3phosphate dehydrogenase was used as an endogenous control to normalize and using the 2- $\Delta \Delta \mathrm{Ct}$ method for lncRNAs expression calculation. The primer sequences were designed in the laboratory based on the DNA sequences and is shown: NONHSAG007503 (forwards primer GGAGAAGTCTGCCGTTAC; reverse primer TCAAAGAACCTCTGGGTCC) and NONHSAT040387 (forwards primer CTTCAGTAGCTCTGCTATGC; reverse primer AGAGTCTGCGTAGTATATGGTA).

\section{Statistical analysis}

All results were represented as the means $\pm \mathrm{SD}$ or proportions. For comparisons, paired t-tests and unpaired t-tests were performed where appropriate. All graphs were plotting using GraphPad Prism 5.0 for Microsoft Windows (GraphPad Software, USA). Two-sided $p$-values were calculated by the SPSS 16.0 (SPSS, USA) and statistical significance was judged when $p<0.05$.

\section{ACKNOWLEDGMENTS}

This work was supported by the Shanghai Committee of Science and Technology (No. 13140903700).

\section{CONFLICTS OF INTEREST}

The authors declare no financial conflicts of interest.

\section{REFERENCES}

1. Luo X, Yang B and Nattel S. MicroRNAs and atrial fibrillation: mechanisms and translational potential. Nature reviews Cardiology. 2014.

2. Dewland TA, Glidden DV and Marcus GM. Healthcare utilization and clinical outcomes after catheter ablation of atrial flutter. PloS one. 2014; 9:e100509.

3. Santulli G, Iaccarino G, De Luca N, Trimarco B and Condorelli G. Atrial fibrillation and microRNAs. Frontiers in physiology. 2014; 5:15.

4. Hung $\mathrm{T}$ and Chang HY. Long noncoding RNA in genome regulation: prospects and mechanisms. RNA biology. 2010; 7:582-585.

5. Di Gesualdo F, Capaccioli S and Lulli M. A pathophysiological view of the long non-coding RNA world. Oncotarget. 2014; 5:10976-10996. doi: 10.18632/ oncotarget. 2770 .

6. Gomes da Silva AM and Silbiger VN. miRNAs as biomarkers of atrial fibrillation. Biomarkers : biochemical indicators of exposure, response, and susceptibility to chemicals. 2014; 19:631-636.

7. Zhao W, Luo J and Jiao S. Comprehensive characterization of cancer subtype associated long non-coding RNAs and their clinical implications. Scientific reports. 2014; 4:6591.

8. Prensner JR and Chinnaiyan AM. The emergence of lncRNAs in cancer biology. Cancer discovery. 2011; 1:391407.

9. Han P, Li W, Lin CH, Yang J, Shang C, Nurnberg ST, Jin $\mathrm{KK}, \mathrm{Xu} \mathrm{W}$, Lin CY, Lin CJ, Xiong Y, Chien HC, Zhou B, Ashley E, Bernstein D, Chen PS, et al. A long noncoding RNA protects the heart from pathological hypertrophy. Nature. 2014; 514:102-106.

10. Papait R, Kunderfranco P, Stirparo GG, Latronico MV and Condorelli G. Long noncoding RNA: a new player of heart failure? Journal of cardiovascular translational research. 2013; 6:876-883.

11. van den Heuvel NH, van Veen TA, Lim B and Jonsson MK. Lessons from the heart: mirroring electrophysiological characteristics during cardiac development to in vitro differentiation of stem cell derived cardiomyocytes. Journal of molecular and cellular cardiology. 2014; 67:12-25.

12. Guttman M and Rinn JL. Modular regulatory principles of large non-coding RNAs. Nature. 2012; 482:339-346.

13. Shi KH, Tao H, Yang JJ, Wu JX, Xu SS and Zhan HY. Role of microRNAs in atrial fibrillation: new insights and perspectives. Cellular signalling. 2013; 25:2079-2084.

14. Ruan Z, Sun X, Sheng H, Zhu L. Long non-coding RNA expression profile in atrial fibrillation. Int J Clin Exp Pathol. 2015; 8:8402-10. 
15. Liu Z, Zhou C, Liu Y, Wang S, Ye P, Miao X and Xia J. The expression levels of plasma micoRNAs in atrial fibrillation patients. PloS one. 2012; 7:e44906.

16. Wang JG, Meng X, Han J, Li Y, Luo TG, Wang J, Xin M and Xi JZ. Differential expressions of miRNAs in patients with nonvalvular atrial fibrillation [Article in Chinese]. Zhonghua yi xue za zhi. 2012; 92:1816-1819.

17. Firouzi M, Bierhuizen MF, Kok B, Teunissen BE, Jansen AT, Jongsma HJ and Groenewegen WA. The human Cx40 promoter polymorphism $-44 \mathrm{G} \longrightarrow$ A differentially affects transcriptional regulation by Sp1 and GATA4. Biochimica et biophysica acta. 2006; 1759:491-496.

18. Gegonne A, Devaiah BN and Singer DS. TAF7: traffic controller in transcription initiation. Transcription. 2013; 4:29-33.

19. Li D, Chen G, Yang J, Fan X, Gong Y, Xu G, Cui Q and Geng B. Transcriptome analysis reveals distinct patterns of long noncoding RNAs in heart and plasma of mice with heart failure. PloS one. 2013; 8:e77938.

20. Zhu JG, Shen YH, Liu HL, Liu M, Shen YQ, Kong XQ, Song GX and Qian LM. Long noncoding RNAs expression profile of the developing mouse heart. Journal of cellular biochemistry. 2014; 115:910-918.

21. Cooley N, Cowley MJ, Lin RC, Marasco S, Wong C, Kaye DM, Dart AM and Woodcock EA. Influence of atrial fibrillation on microRNA expression profiles in left and right atria from patients with valvular heart disease. Physiological genomics. 2012; 44:211-219.

22. Shen X, Xie B, Ma Z, Yu W, Wang W, Xu D, Yan X, Chen B, Yu L, Li J, Chen X, Ding K, Cao F. Identification of novel long non-coding RNAs in triple-negative breast cancer. Oncotarget. 2015; 6:21730-9. doi: 10.18632/ oncotarget. 4419 . 